\title{
Speckle tracking echocardiography in chronic obstructive pulmonary disease and overlapping obstructive sleep apnea
}

This article was published in the following Dove Press journal:

International Journal of COPD

3 August 2016

Number of times this article has been viewed

\section{Carmen Pizarro* \\ Fabian van Essen* \\ Fabian Linnhoff \\ Robert Schueler \\ Christoph Hammerstingl \\ Georg Nickenig \\ Dirk Skowasch \\ Marcel Weber}

Department of Internal Medicine II, Cardiology, Pneumology and Angiology, University Hospital Bonn, Bonn, Germany

*These authors contributed equally to this work
Correspondence: Carmen Pizarro Department of Internal Medicine II, Cardiology, Pneumology and Angiology, University Hospital Bonn, Sigmund-FreudStraße 25, 53I05 Bonn, Germany

Tel +49228 287| 5259

Fax +49228 287I 4323

Email carmen.pizarro@ukb.uni-bonn.de
Background: COPD and congestive heart failure represent two disease entities of growing global burden that share common etiological features. Therefore, we aimed to identify the degree of left ventricular (LV) dysfunction in COPD as a function of COPD severity stages and concurrently placed particular emphasis on the presence of overlapping obstructive sleep apnea (OSA).

Methods: A total of 85 COPD outpatients $(64.1 \pm 10.4$ years, $54.1 \%$ males $)$ and 20 controls, matched for age, sex, and smoking habits, underwent speckle tracking echocardiography for LV longitudinal strain imaging. Complementary 12-lead electrocardiography, laboratory testing, and overnight screening for sleep-disordered breathing using the SOMNOcheck micro ${ }^{\circledR}$ device were performed.

Results: Contrary to conventional echocardiographic parameters, speckle tracking echocardiography revealed significant impairment in global LV strain among COPD patients compared to control smokers $(-13.3 \% \pm 5.4 \%$ vs $-17.1 \% \pm 1.8 \%, P=0.04)$. On a regional level, the apical septal LV strain was reduced in COPD $(P=0.003)$ and associated with the degree of COPD severity $(P=0.02)$. With regard to electrocardiographic findings, COPD patients exhibited a significantly higher mean heart rate than controls $(71.4 \pm 13.0$ beats per minute vs $60.3 \pm 7.7$ beats per minute, $P=0.001$ ) that additionally increased over Global Initiative for Chronic Obstructive Lung Disease stages $(P=0.01)$. Albeit not statistically significant, COPD led to elevated $\mathrm{N}$-terminal pro-brain natriuretic peptide levels $(453.2 \pm 909.0 \mathrm{pg} / \mathrm{mL}$ vs $96.8 \pm 70.0 \mathrm{pg} / \mathrm{mL}, P=0.08)$. As to somnological testing, the portion of COPD patients exhibiting overlapping OSA accounted for $5.9 \%$ and did not significantly vary either in comparison to controls $(P=0.07)$ or throughout the COPD Global Initiative for Chronic Obstructive Lung Disease stages $(P=0.49)$. COPD-OSA overlap solely correlated with nocturnal hypoxemic events, whereas LV performance status was unrelated to coexisting OSA.

Conclusion: To conclude, COPD itself seems to be accompanied with decreased LV deformation properties that worsen over COPD severity stages, but do not vary in case of overlapping OSA. Keywords: chronic obstructive pulmonary disease, speckle tracking echocardiography, left ventricular dysfunction, overlap syndrome

\section{Introduction}

Chronic obstructive pulmonary disease (COPD), characterized by not fully reversible airflow obstruction, imposes a substantial global disease burden. ${ }^{1,2}$ Growing evidence indicates that the natural course of COPD is decisively influenced by associated disorders beyond the lungs. Among these extrapulmonary comorbidities, cardiovascular pathologies constitute major determinants of the disease's morbidity and mortality. ${ }^{3}$ 
This association is even upheld after adjustment for common cardiovascular stressors such as smoking, arterial hypertension, age, or sex and is foremost attributable to persistent low-grade systemic inflammation. ${ }^{4-6}$ Coexistence of COPD and biventricular dysfunction has primarily been ascertained in advanced COPD stages. ${ }^{7}$ It is noteworthy that subclinical biventricular dysfunction is detectable even in mild COPD, suggesting that cardiac comorbidities commence early in COPD and initially remain clinically occult. ${ }^{8}$

Currently, two-dimensional speckle tracking echocardiography (2D STE) constitutes an established noninvasive imaging technique that permits visualization of ventricular strain and analysis of deformation capabilities. ${ }^{9}$ In comparison to conventional echocardiography, STE has shown superior discriminatory power for evaluation of global and regional biventricular function. Kalaycığlu et al recently reported association in COPD between the BODE index (body mass index, airflow obstruction, dyspnea, and exercise capacity) and STE-assessed left ventricular (LV) contractility. ${ }^{10}$

Apart from cardiovascular comorbid conditions, sleepdisordered breathing (SDB) is commonly associated with COPD. Concomitance of obstructive sleep apnea (OSA) and COPD, referred to as "overlap syndrome", constitutes a distinct disease entity that results in profound nocturnal oxygen desaturation and daytime gas exchange derangements. ${ }^{11}$ As repetitive hypoxemic stress enforces blood pressure increases, arrhythmic events, and endothelial dysfunction, ${ }^{12}$ comorbid OSA may exert an additional negative impact on cardiac performance.

Thus, the aim of this prospective cohort study was: 1) to analyze LV performance status by conventional echocardiography and STE, as a function of COPD severity degree, 2) to determine the impact of concomitant OSA on cardiac contractility, and 3 ) to correlate the results with those obtained in matched control smokers.

\section{Methods}

\section{Study population}

Between July 2014 and December 2015, 85/109 consecutive COPD patients under treatment at the outpatient pneumological department of the University Hospital Bonn (Germany) were included in this prospective cohort study. COPD diagnosis was spirometrically and clinically confirmed in accordance with the Global Initiative for Chronic Obstructive Lung Disease (GOLD) diagnostic criteria. ${ }^{2}$ Patients were approached during their outpatient consultation and excluded in case of COPD exacerbation within the preceding 3 months. Patients with previously diagnosed SDB were excluded from study participation.
In addition to the COPD patient population, 20 age- and sex-matched smokers without lung functional abnormalities were selected as controls. Controls were current or former smokers with a smoking history greater than 5 pack-years; their recruitment was carried out from the general population by screening invitation. Both COPD patients and controls underwent overnight screening for SDB and evaluation of cardiac performance status by transthoracic echocardiography, offline LV speckle tracking analysis, and laboratory testing. The study was conducted in compliance with the Declaration of Helsinki and was approved by the medical ethics committee of Bonn (Germany). All participants gave informed written consent before inclusion in the study.

\section{Pulmonary function testing}

Spirometrically determined postbronchodilator forced expiratory volume in one second $\left(\mathrm{FEV}_{1}\right)$, forced vital capacity, and the resulting $\mathrm{FEV}_{1}$ /forced vital capacity ratio $<0.70$ indicated persistent airflow limitation and confirmed COPD. Spirometric degree of obstruction, symptomatology - as assessed by the COPD assessment test - and exacerbation risk defined COPD stages, according to the current guidelines. ${ }^{2}$

\section{Nocturnal SDB screening}

All study participants underwent screening for SDB by a single nighttime outpatient study using SOMNOcheck micro $^{\circledR}$ (Weinmann Medical Technology, Hamburg, Germany). This sleep diagnostic device relies on a twochannel algorithm: by nasal cannula, respiratory flow assessment is achieved; ${ }^{13}$ photoplethysmographic analysis of the pulse wave amplitude enables differentiation between obstructive and central apneic events and evaluation of sleep fragmentation status. ${ }^{14}$ The apnea hypopnea index (AHI) that describes the average number of apneic and hypopneic events per hour of sleep was employed for diagnosing and staging $\mathrm{SDB}^{15}$ : an $\mathrm{AHI}<5 / \mathrm{h}$ excluded SDB, whereas $\mathrm{AHI}$ values of 5 to $<15 / \mathrm{h}, 15$ to $<30 / \mathrm{h}$, and $\geq 30 / \mathrm{h}$ were indicative of mild, moderate, and severe sleep apnea, respectively. Also, the subjects' general daytime sleepiness was quantified by using the Epworth Sleepiness Scale (ESS), with an ESS score $>9$ implying excessive sleepiness. ${ }^{16}$

\section{Electrocardiographic and laboratory testing}

Each study participant underwent 12-lead electrocardiogram and blood sampling that encompassed a full blood count and measurement of plasma N-terminal pro-brain natriuretic peptide (NT-proBNP) levels, which is a cardiac biomarker of myocardial failure. 


\section{Echocardiographic study}

A complete 2D transthoracic echocardiography study was conducted using a $2.5 \mathrm{MHz}$ phased-array transducer and a standard ultrasound scanner (Vivid 7, General Electric Medical Health, Waukesha, WI, USA; iE 33, Philips Medical Systems, Koninklijke NV, Hamburg, Germany), in conformity with the recommendations of the American Society of Echocardiography. ${ }^{17}$ Conventional echocardiographic parameters were measured with the patient in the left lateral decubitus and supine positions for parasternal-apical and subcostal views, respectively. Echocardiographic recordings were digitized and transferred to a commercially available workstation with dedicated software (TomTec Imaging Systems GmbH, Unterschleissheim, Germany) for offline 2D STE analysis. On the basis of a grayscale apical fourchamber view, the LV endocardial border was manually traced, starting from the septal to the lateral mitral annulus, and tracked based on software throughout two cardiac cycles. Regional longitudinal strain assessment was performed by partition of the LV into six standard segments at the apical, midventricular, and basal levels. Global longitudinal LV strain was calculated by averaging the six segmental values. All conventional and STE studies were conducted by experienced cardiologists who were blinded to the patients' pneumological data.

\section{Statistical analysis}

Statistical analyses were conducted using Statistical Package for the Social Sciences 23.0 software (IBM Corporation, Armonk, NY, USA). In case of continuous variables, data were expressed as mean and standard deviation or as median and interquartile range; categorical parameters were presented as frequency and percentage. Comparison of categorical parameters was performed by Fisher's exact test or Pearson's chi-squared test. For continuous variables, Student's $t$-test or univariate analysis of variance was employed. Correlations between variables were examined with Spearman's correlation coefficient. Receiver operating characteristic curve analysis was conducted to describe the predictive value of strain parameters; the correspondent area under the curve with its $95 \%$ confidence interval was calculated. A two-sided $P$-value $<0.05$ was considered statistically significant.

\section{Results}

\section{Clinical characteristics}

We prospectively enrolled 85 COPD outpatients for SDB screening and cardiac performance evaluation, as described above. Demographic characteristics and clinical data of the patient population are summarized in Table 1 . Mean age of the study patients was $64.1 \pm 10.4$ years. Forty-six out of 85 patients $(54.1 \%)$ were males. Staging according to COPD GOLD classification revealed $7.1 \%, 49.4 \%$, $3.5 \%$, and $40.0 \%$ of patients belonging to GOLD groups A-D, respectively. Overall, mean postbronchodilator $\mathrm{FEV}_{1}$ averaged $1.6 \pm 0.81$ in absolute terms and $57.8 \% \pm 22.3 \%$ of the predicted value. As displayed in Table 1, this persistent airflow limitation was accompanied by moderate hyperinflation, restricted diffusion capacity, and impaired oxygenation status that required home oxygen therapy in $17 / 85$ patients.

Twenty controls were matched against COPD patients with respect to age, sex, and smoking history. Neither pack-year defined cumulative smoking status nor current smoking habits differed between groups $(P=0.31$ and $P=0.89$, respectively). However, no airflow obstruction was detected among controls: with the exception of mean partial pressure of carbon dioxide $(P=0.38$ ), pulmonary function values significantly varied between both groups, as did COPD assessment test scores (each with $P<0.001$ ) (Table 1).

\section{Somnological examination}

As to nocturnal respiratory events, SDB screening revealed a mean AHI of $6.5 \pm 7.0 / \mathrm{h}$ within the COPD population, which did not differ from the index obtained in controls (AHI $=5.7 \pm 5.3, P=0.97$ ). Also, comparison of AHI within all five study groups (ie, COPD GOLD stages A through D and controls, the last one termed "COPD GOLD 0") did not offer significant differences $(P=0.47)$. Likewise, no distributive imbalances of primarily obstructive or central apneic events were observed (Table 2). The portion of subjects in whom screening indicated the presence of sleep apnea (by an AHI $\geq 15 / \mathrm{h}$, or an AHI $=5$ to $<15 / \mathrm{h}$ along with an ESS score $\geq 10$ ) was 7 among 85 COPD patients and 3 among 20 controls $(8.2 \%$ vs $15.0 \%, P=0.81)$. Concomitant OSA in COPD patients - referred to as overlap syndrome - was observed in $5 / 85$ patients (5.9\%); however, the prevalence of OSA was balanced between COPD patients and controls (5.9\% vs $5.0 \%, P=0.07$ ), as well as throughout the distinct COPD GOLD severity groups $(P=0.49)$.

With regard to nocturnal oxygenation status, mean oxygen saturation correlated inversely with COPD GOLD stages (Spearman's rho: $-0.40, P=0.04$ ) and was significantly lower in COPD patients than among controls $(93.5 \% \pm 2.0 \%$ vs $95.4 \% \pm 0.8 \%, P=0.003$ ). By contrast, no distributive differences were ascertained either for the oxygen desaturation index (ODI) or for the minimum levels of oxygen saturation recorded (Table 2). 
Table I Clinical data of study participants as a function of presence and severity of COPD

\begin{tabular}{|c|c|c|c|c|c|c|c|c|}
\hline Categorical variables & $\begin{array}{l}\text { COPD } \\
\text { GOLD A }\end{array}$ & $\begin{array}{l}\text { COPD } \\
\text { GOLD B }\end{array}$ & $\begin{array}{l}\text { COPD } \\
\text { GOLD C }\end{array}$ & $\begin{array}{l}\text { COPD } \\
\text { GOLD D }\end{array}$ & $\begin{array}{l}\text { All COPD } \\
\text { patients }\end{array}$ & Controls & $\begin{array}{l}\text { All study } \\
\text { participants }\end{array}$ & $P$-value \\
\hline Subjects, n (\%) & $6(5.7)$ & $42(40.0)$ & $3(2.9)$ & $34(32.4)$ & $85(81.0)$ & $20(19.0)$ & $105(100.0)$ & \\
\hline \multicolumn{9}{|l|}{ Demographics } \\
\hline Age (years) & $65.3 \pm 10.1$ & $62.8 \pm 11.5$ & $73.7 \pm 5.1$ & $64.7 \pm 9.1$ & $64.1 \pm 10.4$ & $61.3 \pm 6.9$ & $63.6 \pm 9.9$ & 0.27 \\
\hline Male, n (\%) & $3(50.0)$ & $25(59.5)$ & I (33.3) & $17(50.0)$ & $46(54.1)$ & II (55.0) & $57(54.3)$ & 0.94 \\
\hline BMI $\left(\mathrm{kg} / \mathrm{m}^{2}\right)$ & $26.8 \pm 6.0$ & $26.2 \pm 5.0$ & $23.6 \pm 3.5$ & $24.8 \pm 5.8$ & $25.9 \pm 5.4$ & $27.8 \pm 4.1$ & $26.3 \pm 5.2$ & 0.21 \\
\hline CAT & $8.0 \pm 2.0$ & $20.3 \pm 5.7$ & $6.54 \pm 3.5$ & $22.9 \pm 6.2$ & $20.3 \pm 7.0$ & $8.4 \pm 5.7$ & $17.7 \pm 8.3$ & $<0.001$ \\
\hline \multicolumn{9}{|l|}{ Pulmonary function parameters ${ }^{a}$} \\
\hline $\mathrm{FEV}_{1}(\mathrm{~L})$ & $1.97 \pm 0.73$ & $2.07 \pm 0.8 \mathrm{I}$ & $1.07 \pm 0.21$ & $1.07 \pm 0.40$ & $\mathrm{I} .6 \mathrm{I} \pm 0.82$ & $2.90 \pm 0.86$ & $1.86 \pm 0.97$ & $<0.001$ \\
\hline FEV $(\%$ predicted) & $77.2 \pm 19.8$ & $70.9 \pm 17.5$ & $46.1 \pm 3.9$ & $39.3 \pm 13.3$ & $57.8 \pm 22.3$ & $98.1 \pm 13.5$ & $65.6 \pm 26.3$ & $<0.001$ \\
\hline $\mathrm{FEV}_{1} / \mathrm{FVC}$ ratio & $63.1 \pm 11.0$ & $67.9 \pm 11.2$ & $58.6 \pm 13.1$ & $52.4 \pm \mid 4.3$ & $60.5 \pm 15.1$ & $78.6 \pm 7.3$ & $64.0 \pm 15.6$ & $<0.001$ \\
\hline $\mathrm{RV}(\mathrm{L})$ & $3.76 \pm 1.40$ & $2.93 \pm 0.96$ & $3.58 \pm 1.20$ & $4.26 \pm 1.24$ & $3.82 \pm 1.52$ & $2.53 \pm 0.94$ & $3.56 \pm I .5 I$ & $<0.001$ \\
\hline RV (\% predicted) & $166.1 \pm 50.4$ & $130.6 \pm 44.5$ & $|4| .7 \pm 23.5$ & $181.7 \pm 43.5$ & $|7| .0 \pm 67.9$ & $111.4 \pm 35.9$ & $159.6 \pm 66.9$ & $<0.001$ \\
\hline $\mathrm{R}$ tot $(\mathrm{kPa} s / \mathrm{L})$ & $0.35 \pm 0.14$ & $0.30 \pm 0.14$ & $0.40 \pm 0.03$ & $0.50 \pm 0.18$ & $0.46 \pm 0.27$ & $0.21 \pm 0.08$ & $0.42 \pm 0.26$ & $<0.001$ \\
\hline $\mathrm{R}$ tot (\% predicted) & $115.4 \pm 46.5$ & $100.9 \pm 46.2$ & $132.7 \pm 9.7$ & $165.5 \pm 59.4$ & $155.3 \pm 92.0$ & $71.6 \pm 27.5$ & $140.4 \pm 89.3$ & $<0.001$ \\
\hline DLco (\% predicted) & $74.9 \pm 8.2$ & $54.7 \pm 11.8$ & $47.7 \pm 15.3$ & $34.9 \pm 15.5$ & $48.5 \pm 17.1$ & $78.3 \pm 12.6$ & $57.1 \pm 20.7$ & $<0.001$ \\
\hline DLco/VA (\% predicted) & $88.2 \pm 7.1$ & $70.0 \pm 14.2$ & $70.2 \pm 18.5$ & $45.1 \pm 16.9$ & $60.9 \pm 21.5$ & $91.7 \pm 15.5$ & $69.5 \pm 24.0$ & $<0.001$ \\
\hline \multicolumn{9}{|l|}{ Capillary blood gas analysis } \\
\hline $\mathrm{pO}_{2}(\mathrm{mmHg})$ & $70.1 \pm 5.6$ & $65.5 \pm 9.5$ & $64.0 \pm 3.0$ & $59.1 \pm 6.9$ & $66.4 \pm 13.3$ & $77.6 \pm 4.3$ & $68.5 \pm 12.9$ & $<0.001$ \\
\hline $\mathrm{PCO}_{2}(\mathrm{mmHg})$ & $33.5 \pm 6.2$ & $35.1 \pm 8.0$ & $37.4 \pm 0.8$ & $37.5 \pm 3.9$ & $36.3 \pm 6.4$ & $34.9 \pm 4.8$ & $36.0 \pm 6.1$ & 0.38 \\
\hline $\mathrm{sO}_{2}(\%)$ & $95.7 \pm 0.7$ & $93.9 \pm 3.8$ & $94.0 \pm 2.0$ & $91.2 \pm 2.6$ & $93.5 \pm 2.9$ & $96.3 \pm 0.8$ & $94.1 \pm 2.8$ & $<0.001$ \\
\hline \multicolumn{9}{|l|}{ Cardiovascular risk factors, $n$ (\%) } \\
\hline Arterial hypertension & $5(83.3)$ & $19(45.2)$ & $2(66.7)$ & $23(67.6)$ & $49(57.6)$ & $10(50.0)$ & $59(56.2)$ & 0.47 \\
\hline Diabetes mellitus & $0(0)$ & $6(14.3)$ & $0(0)$ & $6(17.6)$ & $12(14.1)$ & $2(10.0)$ & $14(13.3)$ & 0.53 \\
\hline Dyslipidemia & $2(33.3)$ & $18(42.9)$ & $2(66.7)$ & II (32.4) & $33(38.8)$ & $7(35.0)$ & $40(38.1)$ & 0.53 \\
\hline Familial disposition & $2(33.3)$ & $15(35.7)$ & $0(0)$ & $6(17.6)$ & $23(27.1)$ & $10(50.0)$ & $33(31.4)$ & 0.33 \\
\hline \multicolumn{9}{|l|}{ Smoking habits } \\
\hline Current smoker, n (\%) & $0(0)$ & $10(23.8)$ & I (33.3) & $6(17.6)$ & $17(20.0)$ & $4(20.0)$ & $21(20.0)$ & 0.89 \\
\hline Former smoker, n (\%) & $5(83.3)$ & $29(69.0)$ & $2(66.7)$ & $27(79.4)$ & $63(74.1)$ & $16(80.0)$ & $79(75.2)$ & \\
\hline Never smoker, n (\%) & I (16.7) & $3(7.1)$ & $0(0)$ & I (2.9) & $5(5.9)$ & $0(0)$ & $5(4.8)$ & \\
\hline Pack-years, median (range) & $10(0-20)$ & $30(0-125)$ & $40(20-60)$ & $40(0-80)$ & $30(0-125)$ & $30(5-150)$ & $30(0-150)$ & 0.31 \\
\hline Alpha-I antitrypsin deficiency, $\mathrm{n}(\%)$ & $0(0)$ & $2(4.8)$ & I (33.3) & $2(5.9)$ & $5(5.9)$ & $0(0)$ & $5(4.8)$ & 0.26 \\
\hline \multicolumn{9}{|l|}{ Prediagnosed comorbidities, n (\%) } \\
\hline Pulmonary hypertension & $0(0)$ & $6(14.3)$ & I (33.3) & $5(14.7)$ & $12(14.1)$ & $0(0)$ & $12(11.4)$ & 0.07 \\
\hline Coronary heart disease & $4(66.7)$ & $13(31.0)$ & I (33.3) & $6(17.6)$ & $24(28.2)$ & $2(10.0)$ & $26(24.8)$ & 0.09 \\
\hline \multicolumn{9}{|c|}{ Antiobstructive and anti-inflammatory medication use, $\mathbf{n}(\%)$} \\
\hline Long-acting beta ${ }_{2}$ agonist & $0(0)$ & $29(69.0)$ & $3(100.0)$ & $28(82.4)$ & $60(70.6)$ & $0(0)$ & $60(57.1)$ & $<0.001$ \\
\hline Long-acting anticholinergic & $0(0)$ & $22(52.4)$ & I (33.3) & $28(82.4)$ & $51(60.0)$ & $0(0)$ & $51(48.6)$ & $<0.001$ \\
\hline Inhaled glucocorticoid & $0(0)$ & $0(0)$ & $2(66.7)$ & $27(79.4)$ & $29(34.1)$ & $0(0)$ & $29(27.6)$ & $<0.001$ \\
\hline Systemic glucocorticoid & $0(0)$ & $0(0)$ & $0(0)$ & $4(I I .8)$ & $4(4.7)$ & $0(0)$ & $4(3.8)$ & 0.003 \\
\hline PDE-4 inhibitor (roflumilast) & $0(0)$ & $0(0)$ & I (33.3) & $14(4 \mid .2)$ & $15(17.6)$ & $0(0)$ & $15(14.3)$ & $<0.001$ \\
\hline Home oxygen therapy, n (\%) & $0(0)$ & $0(0)$ & $2(66.7)$ & I5 (44.I) & $17(20.0)$ & $0(0)$ & $17(16.2)$ & 0.03 \\
\hline
\end{tabular}

Notes: Data are presented as mean \pm standard deviation, median (range), or $\mathrm{n}$ (\%). P-value refers to data comparison between the entire COPD group and controls. ${ }^{3}$ Based on postbronchodilator pulmonary function testing.

Abbreviations: BMI, body mass index; CAT, COPD assessment test; DLco, diffusion capacity for carbon monoxide; FEV , forced expiratory volume in I second; FVC, forced vital capacity; GOLD, Global initiative for Chronic Obstructive Lung Disease; $\mathrm{PCO}_{2}$, carbon dioxide partial pressure; $\mathrm{PDE}-4$, phosphodiesterase type 4; $\mathrm{pO}{ }_{2}$, oxygen partial pressure; $\mathrm{R}$ tot, total resistance; $\mathrm{RV}$, residual volume; $\mathrm{sO}_{2}$, oxygen saturation; $\mathrm{VA}$, alveolar volume.

As aforementioned, 5/85 COPD patients suffered from overlapping OSA. Within the COPD cohort, presence of overlap syndrome was related to higher nocturnal ODIs (12.2 $\pm 14.4 / \mathrm{h}$ vs $2.8 \pm 3.4 / \mathrm{h}, P=0.001$ ) (Figure 1). In keeping with this, minimal nocturnal oxygen saturation was significantly lower in overlap patients than in COPD patients without concomitant OSA $(79.8 \% \pm 3.3 \%$ vs $84.8 \% \pm 5.9 \%$,
$P=0.02)$. However, pulmonary function parameters did not differ between groups.

\section{Cardiological examination}

Echocardiographic, electrocardiographic, and laboratory parameters are provided in Table 3. Conventional echocardiography showed an overall preserved systolic LV function 
Table 2 Results obtained by screening for sleep-disordered breathing as a function of presence and severity of COPD

\begin{tabular}{|c|c|c|c|c|c|c|c|c|c|}
\hline $\begin{array}{l}\text { Categorical } \\
\text { variables }\end{array}$ & $\begin{array}{l}\text { COPD } \\
\text { GOLD A }\end{array}$ & $\begin{array}{l}\text { COPD } \\
\text { GOLD B }\end{array}$ & $\begin{array}{l}\text { COPD } \\
\text { GOLD C }\end{array}$ & $\begin{array}{l}\text { COPD } \\
\text { GOLD D }\end{array}$ & $\begin{array}{l}\text { All COPD } \\
\text { patients }\end{array}$ & Controls & $\begin{array}{l}\text { All study } \\
\text { participants }\end{array}$ & $\begin{array}{l}P \text {-value }{ }^{\text {a }} \text { (COPD } \\
\text { group vs } \\
\text { controls) }\end{array}$ & $\begin{array}{l}P \text {-value }{ }^{\mathrm{b}} \text { (COPD } \\
\text { GOLD A through } \\
\text { D and controls) }\end{array}$ \\
\hline Subjects, n (\%) & $6(5.7)$ & $42(40.0)$ & $3(2.9)$ & $34(32.4)$ & $85(81.0)$ & $20(19.0)$ & $105(100.0)$ & & \\
\hline Recording time (h) & $6.05 \pm 2.16$ & $5.63 \pm 1.27$ & $6.95 \pm 0.92$ & $7.12 \pm 0.70$ & $6.19 \pm 1.50$ & $6.38 \pm 1.18$ & $6.29 \pm 1.42$ & 0.38 & 0.42 \\
\hline \multicolumn{10}{|c|}{ Respiratory events (per h) } \\
\hline $\mathrm{AHI}$ & $5.8 \pm 3.2$ & $5.37 \pm 6.53$ & $9.35 \pm 1.06$ & $2.06 \pm 1.40$ & $6.48 \pm 7.01$ & $5.74 \pm 5.26$ & $6.47 \pm 6.65$ & 0.97 & 0.47 \\
\hline Obstructive AHI & $3.6 \pm 3.0$ & $2.57 \pm 3.91$ & $5.75 \pm 1.91$ & $0.86 \pm 0.83$ & $3.56 \pm 5.04$ & $2.93 \pm 4.57$ & $3.33 \pm 4.79$ & 0.56 & 0.55 \\
\hline Central AHI & $1.9 \pm 0.9$ & $2.46 \pm 2.89$ & $2.60 \pm 2.40$ & $1.16 \pm 0.71$ & $2.20 \pm 2.08$ & $2.63 \pm 2.06$ & $2.32 \pm 2.04$ & 0.45 & 0.63 \\
\hline \multicolumn{10}{|c|}{ Autonomic arousals (per $h$ ) } \\
\hline $\begin{array}{l}\text { Autonomic arousal } \\
\text { index }\end{array}$ & $9.6 \pm 9.0$ & $|5.1 \pm| 3 . \mid$ & II. $3 \pm 4.4$ & $|8.1 \pm 1| .3$ & $16.9 \pm 12.9$ & $15.8 \pm 10.2$ & $16.8 \pm 12.0$ & 0.93 & 0.71 \\
\hline $\begin{array}{l}\text { Respiratory arousal } \\
\text { index }\end{array}$ & $1.1 \pm 0.9$ & $2.3 \pm 3.6$ & $3.1 \pm 1.7$ & $0.9 \pm 0.4$ & $3.1 \pm 4.1$ & $2.5 \pm 1.9$ & $2.9 \pm 3.7$ & 0.65 & 0.34 \\
\hline $\begin{array}{l}\text { Respiratory effort } \\
\text { related arousal index }\end{array}$ & $0.33 \pm 0.35$ & $0.53 \pm 0.75$ & $1.50 \pm 0.42$ & $0.18 \pm 0.25$ & $0.91 \pm 1.44$ & $0.57 \pm 0.85$ & $0.87 \pm 1.33$ & 0.71 & 0.56 \\
\hline \multicolumn{10}{|l|}{ Nocturnal oxygenation } \\
\hline ODI (per h) & $4.0 \pm 3.9$ & $5.5 \pm 9.9$ & $4.7 \pm 1.9$ & $1.5 \pm 2.0$ & $3.7 \pm 6.1$ & $1.4 \pm 1.1$ & $3.3 \pm 5.4$ & 0.31 & 0.45 \\
\hline Minimal saturation (\%) & $85.5 \pm 3.7$ & $83.8 \pm 8.9$ & $80.0 \pm 2.8$ & $86.8 \pm 3.2$ & $84.0 \pm 6.1$ & $87.6 \pm 4.8$ & $84.8 \pm 5.8$ & 0.06 & 0.32 \\
\hline Mean saturation (\%) & $93.8 \pm 1.5$ & $94.4 \pm 2.0$ & $93.0 \pm 1.4$ & $93.4 \pm 0.9$ & $93.5 \pm 2.0$ & $95.4 \pm 0.8$ & $93.9 \pm 2.0$ & 0.003 & 0.04 \\
\hline $\begin{array}{l}\text { Nocturnal heart rate } \\
\text { (beats per minute) }\end{array}$ & $68.3 \pm 5.3$ & $65.7 \pm 6.5$ & $70.0 \pm 1.4$ & $70.9 \pm 9.1$ & $67.5 \pm 7.3$ & $60.0 \pm 6.0$ & $65.7 \pm 7.7$ & 0.001 & 0.003 \\
\hline \multicolumn{10}{|c|}{ Epworth sleepiness scale } \\
\hline Mean score & $2.0 \pm 1.4$ & $7.9 \pm 5.2$ & $5.0 \pm 4.2$ & $5.7 \pm 3.5$ & $6.5 \pm 4.6$ & $7.5 \pm 5.1$ & $6.7 \pm 4.7$ & 0.48 & 0.06 \\
\hline Score $\geq 10, \mathrm{n}(\%)$ & $0(0)$ & $10(23.8)$ & $0(0)$ & $3(8.8)$ & $13(15.3)$ & $2(10.0)$ & $15(14.3)$ & 0.39 & 0.22 \\
\hline
\end{tabular}

Notes: Data are presented as mean \pm standard deviation or $\mathrm{n}(\%)$. ${ }^{\mathrm{a}} \mathrm{P}$-value refers to data comparison between the entire COPD group and controls. ${ }^{\mathrm{b}} \mathrm{P}$-value refers to data comparison between all five groups (COPD GOLD A through D and controls).

Abbreviations: h, hour; AHI, apnea hypopnea index; GOLD, Global initiative for Chronic Obstructive Lung Disease; ODI, oxygen desaturation index.

without differences between groups. Likewise, diastolic LV performance and right functional echocardiographic parameters - such as pulmonary arterial systolic pressure and tricuspid annular plane systolic excursion - lacked significant intercohortal differences. On the contrary, STE-based longitudinal LV performance evaluation revealed a relevantly

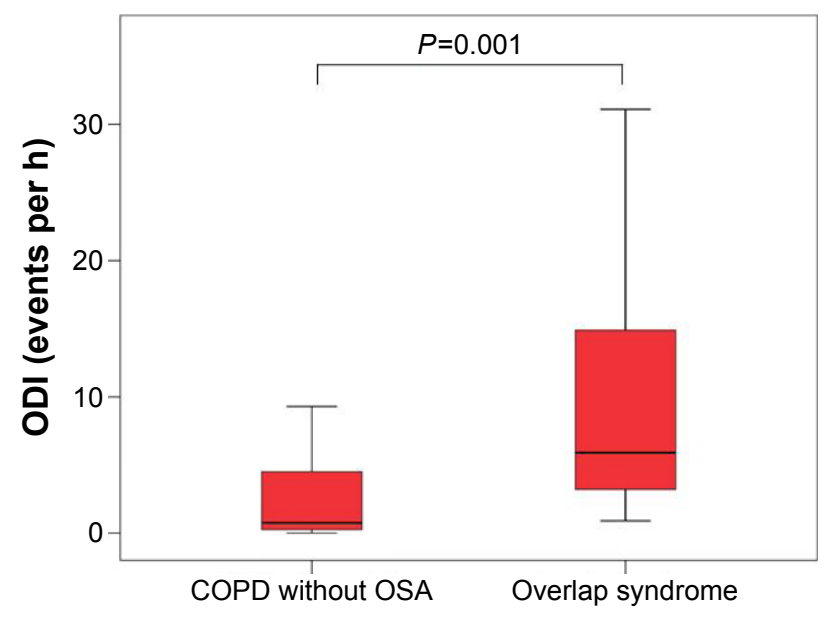

Figure I ODI, stratified by the presence of overlapping OSA in COPD patients. Abbreviations: h, hour; ODI, oxygen desaturation index; OSA, obstructive sleep apnea. impaired mean global LV strain in the total COPD cohort compared to controls $(-13.3 \% \pm 5.4 \%$ vs $-17.1 \% \pm 1.8 \%$, $P=0.04$ ) (Figures $2 \mathrm{~A}, \mathrm{~B}$ and $3 \mathrm{~A}$ ). Additional statistically significant relations were observed on linear regression analysis between global LV strain and FEV1 of the predicted value (Spearman's rho: $-0.26, P=0.04$ ). On a regional level, the apical septal LV strain was significantly reduced in COPD patients $(P=0.003)$ (Figure 3B) and was associated with the severity degree of COPD $(P=0.02)$. Receiver operating characteristic curve analysis reinforced the predicted value of the apical septal strain for identification of patients with COPD (area under the curve: $0.81 ; 95 \%$ confidence interval: 0.67-0.95; $P=0.003$ ) (Figure 4).

With regard to electrocardiographic findings, COPD patients exhibited a significantly higher mean heart rate than controls ( $71.4 \pm 13.0$ beats per minute vs $60.3 \pm 7.7$ beats per minute, $P=0.001)$; moreover, the heart rate increased within COPD stages $(P=0.01)$. This observation is consistent with the results derived from somnological screening: nocturnal heart rate accelerated over COPD severity degrees $(P=0.003)$ and significantly exceeded the heart rate of controls $(P=0.001)$ (Table 2; Figure 5A and $\mathrm{B}$ ). 
Table 3 Comparison of laboratory, electrocardiographic, as well as conventional and speckle tracking-based echocardiographic data according to presence and severity of COPD

\begin{tabular}{|c|c|c|c|c|c|c|c|c|c|}
\hline $\begin{array}{l}\text { Categorical } \\
\text { variables }\end{array}$ & $\begin{array}{l}\text { COPD } \\
\text { GOLD A }\end{array}$ & $\begin{array}{l}\text { COPD } \\
\text { GOLD B }\end{array}$ & $\begin{array}{l}\text { COPD } \\
\text { GOLD C }\end{array}$ & $\begin{array}{l}\text { COPD } \\
\text { GOLD D }\end{array}$ & $\begin{array}{l}\text { All COPD } \\
\text { patients }\end{array}$ & Controls & $\begin{array}{l}\text { All study } \\
\text { participants }\end{array}$ & $\begin{array}{l}P \text {-value } \\
\text { (COPD group } \\
\text { vs controls) }\end{array}$ & $\begin{array}{l}P \text {-value } \\
\text { (COPD GOLD } \\
\text { A through D } \\
\text { and controls) }\end{array}$ \\
\hline Subjects, n (\%) & $6(5.7)$ & $42(40.0)$ & $3(2.9)$ & $34(32.4)$ & $85(81.0)$ & $20(19.0)$ & $105(100.0)$ & & \\
\hline \multicolumn{10}{|c|}{ Laboratory testing } \\
\hline $\begin{array}{l}\text { Leukocytes } \\
\left(10^{\%} / \mathrm{mL}\right)\end{array}$ & $7.8 \pm 3.7$ & $9.2 \pm 3.0$ & $12.7 \pm 8.6$ & $9.7 \pm 3.3$ & $9.4 \pm 3.3$ & $6.9 \pm 2.3$ & $8.9 \pm 3.3$ & 0.002 & 0.02 \\
\hline $\begin{array}{l}\text { Hemoglobin } \\
(\mathrm{g} / \mathrm{dL})\end{array}$ & $14.7 \pm 1.6$ & $14.2 \pm 1.6$ & $14.5 \pm 1.9$ & $13.8 \pm 1.8$ & $14.1 \pm 1.6$ & $14.5 \pm 1.5$ & $14.2 \pm 1.6$ & 0.32 & 0.65 \\
\hline $\begin{array}{l}\text { Thrombocytes } \\
\left(10^{9} / \mathrm{mL}\right)\end{array}$ & $228.6 \pm 45.2$ & $251.5 \pm 60.5$ & $208.5 \pm 23.3$ & $270.7 \pm 90.0$ & $256.5 \pm 71.9$ & $252.6 \pm 44.0$ & $255.7 \pm 66.9$ & 0.82 & 0.58 \\
\hline $\begin{array}{l}\text { NT-proBNP } \\
(\mathrm{pg} / \mathrm{mL})\end{array}$ & $228.0 \pm 151.4$ & $478.8 \pm 911.1$ & $178.5 \pm 173.2$ & $478.3 \pm 1,013.6$ & $453.2 \pm 909.0$ & $96.8 \pm 70.0$ & $384.0 \pm 827.8$ & 0.08 & 0.45 \\
\hline \multicolumn{10}{|c|}{ Electrocardiographic parameters, n (\%) } \\
\hline $\begin{array}{l}\text { Heart rate (beats } \\
\text { per minute) }\end{array}$ & $65.2 \pm 6.7$ & $71.1 \pm 12.6$ & $67.0 \pm 5.7$ & $73.2 \pm 14.5$ & $71.4 \pm 13.0$ & $60.3 \pm 7.7$ & $69.2 \pm 12.9$ & 0.001 & 0.01 \\
\hline \multicolumn{10}{|l|}{ Rhythm, n (\%) } \\
\hline Sinus rhythmus & $6(100.0)$ & $4 \mathrm{I}(97.6)$ & $3(100.0)$ & $34(100.0)$ & $84(98.8)$ & $20(100.0)$ & $104(99.0)$ & 0.62 & 0.87 \\
\hline Atrial fibrillation & $0(0)$ & I (2.4) & $0(0)$ & $0(0)$ & $\mathrm{I}(\mathrm{I} .2)$ & $0(0)$ & $\mathrm{I}(\mathrm{I} .0)$ & & \\
\hline \multicolumn{10}{|c|}{ Premature beats, n (\%) } \\
\hline Atrial & $0(0)$ & $0(0)$ & $0(0)$ & $0(0)$ & $0(0)$ & I (5.0) & $\mathrm{I}(\mathrm{I} .0)$ & 0.04 & 0.39 \\
\hline Ventricular & $0(0)$ & $6(14.3)$ & $0(0)$ & $3(8.8)$ & $9(10.6)$ & $0(0)$ & $9(8.6)$ & 0.12 & 0.35 \\
\hline \multicolumn{10}{|c|}{ Bundle branch block, n (\%) } \\
\hline $\begin{array}{l}\text { No bundle } \\
\text { branch block }\end{array}$ & $6(100.0)$ & $38(90.5)$ & $2(66.7)$ & $27(79.4)$ & $73(85.9)$ & $19(95.0)$ & $92(87.6)$ & $0.28^{c}$ & $0.03^{c}$ \\
\hline $\begin{array}{l}\text { Right bundle } \\
\text { branch block }\end{array}$ & $0(0)$ & $3(7.1)$ & I (33.3) & $7(20.6)$ & II (I2.9) & I (5.0) & $12(11.4)$ & & \\
\hline $\begin{array}{l}\text { Left bundle } \\
\text { branch block }\end{array}$ & $0(0)$ & $\mathrm{I}(2.4)$ & $0(0)$ & $0(0)$ & $\mathrm{I}(\mathrm{I} .2)$ & $0(0)$ & $\mathrm{I}(\mathrm{I} .0)$ & & \\
\hline \multicolumn{10}{|c|}{ Conventional echocardiographic parameters } \\
\hline $\begin{array}{l}\text { LV ejection } \\
\text { fraction (\%) }\end{array}$ & $62.5 \pm 7.1$ & $61.9 \pm 9.5$ & $68.8 \pm 5.4$ & $61.0 \pm 8.9$ & $61.7 \pm 9.0$ & $63.5 \pm 4.5$ & $62.1 \pm 8.3$ & 0.41 & 0.67 \\
\hline \multicolumn{10}{|c|}{ Diastolic dysfunction, n (\%) } \\
\hline $\begin{array}{l}\text { No diastolic } \\
\text { dysfunction }\end{array}$ & $4(66.7)$ & $19(45.2)$ & I (33.3) & $6(17.6)$ & $30(35.3)$ & $7(35.0)$ & $37(35.2)$ & 0.78 & 0.18 \\
\hline Grade I & $2(33.3)$ & $21(50.0)$ & I (33.3) & $27(79.4)$ & $51(60.0)$ & II (55.0) & $62(59.0)$ & & \\
\hline Grade II & $0(0)$ & $2(4.8)$ & I (33.3) & I (2.9) & $4(4.7)$ & $2(10.0)$ & $6(5.7)$ & & \\
\hline Grade III & $0(0)$ & $0(0)$ & $0(0)$ & $0(0)$ & $0(0)$ & $0(0)$ & $0(0)$ & & \\
\hline PASP (mmHg) & $23.2 \pm 9.8$ & $30.2 \pm 11.1$ & $26.5 \pm 3.6$ & $34.5 \pm 17.3$ & $31.0 \pm 13.7$ & $30.0 \pm 4.3$ & $30.9 \pm 13.1$ & 0.90 & 0.71 \\
\hline TAPSE $(\mathrm{cm})$ & $2.50 \pm 0.5 \mathrm{I}$ & $1.94 \pm 0.58$ & $2.20 \pm 0.28$ & $2.09 \pm 0.57$ & $2.07 \pm 0.56$ & $2.48 \pm 0.54$ & $2.13 \pm 0.57$ & 0.14 & 0.32 \\
\hline $\begin{array}{l}\text { Mitral valve } \\
\text { disease, } \mathbf{n}(\%)\end{array}$ & $2(33.3)$ & $25(59.5)$ & I (33.3) & $21(61.8)$ & $49(57.6)$ & $15(75.0)$ & $64(61.0)$ & 0.42 & 0.72 \\
\hline $\begin{array}{l}\text { Aortic valve } \\
\text { disease, } n \text { (\%) }\end{array}$ & I (16.7) & $12(28.6)$ & I (33.3) & $6(17.6)$ & $20(23.5)$ & $3(15.0)$ & $23(21.9)$ & 0.31 & 0.78 \\
\hline $\begin{array}{l}\text { Tricuspid valve } \\
\text { disease, n (\%) }\end{array}$ & I (I6.7) & $17(40.5)$ & $2(66.7)$ & $19(55.9)$ & $39(45.9)$ & $15(75.0)$ & $54(51.4)$ & 0.18 & 0.89 \\
\hline \multicolumn{10}{|c|}{ STE-based LV longitudinal strain assessment } \\
\hline $\begin{array}{l}\text { Apical lateral } \\
\text { strain (\%) }\end{array}$ & $-13.7 \pm 4.6$ & $-11.9 \pm 7.0$ & $-9.1 \pm 8.8$ & $-11.1 \pm 5.9$ & $-11.5 \pm 6.5$ & $-16.2 \pm 5.3$ & $-12.3 \pm 6.5$ & 0.04 & 0.31 \\
\hline $\begin{array}{l}\text { Medial lateral } \\
\text { strain (\%) }\end{array}$ & $-12.8 \pm 7.1$ & $-11.8 \pm 4.8$ & $-14.2 \pm 3.4$ & $-11.8 \pm 3.8$ & $-11.9 \pm 4.4$ & $-13.6 \pm 3.9$ & $-12.2 \pm 4.3$ & 0.33 & 0.81 \\
\hline $\begin{array}{l}\text { Basal lateral } \\
\text { strain (\%) }\end{array}$ & $-12.8 \pm 4.2$ & $-17.3 \pm 7.3$ & $-\mid 7.1 \pm I I . I$ & $-15.0 \pm 6.5$ & $-16.2 \pm 7.0$ & $-17.0 \pm 6.1$ & $-16.4 \pm 6.8$ & 0.75 & 0.78 \\
\hline $\begin{array}{l}\text { Apical septal } \\
\text { strain (\%) }\end{array}$ & $-|8.2 \pm 4|$. & $-16.4 \pm 8.4$ & $-14.5 \pm 13.6$ & $-21.1 \pm 9.6$ & $-18.1 \pm 9.1$ & $-27.9 \pm 6.8$ & $-19.6 \pm 9.4$ & 0.003 & 0.02 \\
\hline $\begin{array}{l}\text { Medial septal } \\
\text { strain (\%) }\end{array}$ & $-13.2 \pm 3.9$ & $-13.3 \pm 5.8$ & $-14.0 \pm 1.9$ & $-13.7 \pm 6.1$ & $-13.5 \pm 5.7$ & $-13.1 \pm 5.5$ & $-13.4 \pm 5.6$ & 0.88 & 0.99 \\
\hline
\end{tabular}


Table 3 (Continued)

\begin{tabular}{|c|c|c|c|c|c|c|c|c|c|}
\hline $\begin{array}{l}\text { Categorical } \\
\text { variables }\end{array}$ & $\begin{array}{l}\text { COPD } \\
\text { GOLD A }\end{array}$ & $\begin{array}{l}\text { COPD } \\
\text { GOLD B }\end{array}$ & $\begin{array}{l}\text { COPD } \\
\text { GOLD C }\end{array}$ & $\begin{array}{l}\text { COPD } \\
\text { GOLD D }\end{array}$ & $\begin{array}{l}\text { All COPD } \\
\text { patients }\end{array}$ & Controls & $\begin{array}{l}\text { All study } \\
\text { participants }\end{array}$ & $\begin{array}{l}P \text {-value }^{a} \\
\text { (COPD group } \\
\text { vs controls) }\end{array}$ & $\begin{array}{l}P \text {-value } \\
\text { (COPD GOLD } \\
\text { A through D } \\
\text { and controls) }\end{array}$ \\
\hline $\begin{array}{l}\text { Basal septal } \\
\text { strain (\%) }\end{array}$ & $-17.8 \pm 3.7$ & $-10.6 \pm 6.9$ & $-9.1 \pm 4.0$ & $-10.7 \pm 5.7$ & $-10.8 \pm 6.3$ & $-10.0 \pm 3.7$ & $-10.6 \pm 5.9$ & 0.72 & 0.81 \\
\hline Global strain (\%) & $-13.9 \pm 1.1$ & $-13.8 \pm 3.4$ & $-17.2 \pm 2.3$ & $-12.0 \pm 7.7$ & $-13.3 \pm 5.4$ & $-17.1 \pm 1.8$ & $-13.9 \pm 5.2$ & 0.04 & 0.14 \\
\hline
\end{tabular}

Notes: Data are presented as mean \pm standard deviation or $\mathrm{n}(\%) .{ }^{a}$-value refers to data comparison between the entire COPD group and controls. ${ }^{b} \mathrm{P}$-value refers to data comparison between all five groups (COPD GOLD A through D and controls). ${ }^{c} P$-value refers to data comparison for occurrence of right bundle branch block. ${ }^{\mathrm{d}}$ Comprises both complete and incomplete bundle branch block morphology.

Abbreviations: GOLD, Global initiative for Chronic Obstructive Lung Disease; LV, left ventricular; NT-proBNP, N-terminal pro-brain natriuretic peptide; PASP, pulmonary arterial systolic pressure; STE, speckle tracking echocardiography; TAPSE, tricuspid annular plane systolic excursion.

Laboratory testing evidenced elevated NT-proBNP levels among COPD patients as compared to controls $(453.2 \pm 909.0$ $\mathrm{pg} / \mathrm{mL}$ vs $96.8 \pm 70.0 \mathrm{pg} / \mathrm{mL})$; however, NT-proBNP distribution failed to reach statistical significance $(P=0.08)$. Nonetheless, linear regression analysis revealed significant relation between NT-proBNP levels and the following postbronchodilator pulmonary function and capillary blood gas parameters: $\mathrm{FEV}_{1} \%$ predicted $(P=0.03)$, total airway resistance \% predicted $(P=0.04)$, oxygen partial pressure $(P=0.004)$, and oxygen saturation $(P=0.001)$.

\section{Discussion}

In a prospective analysis of a cohort of $85 \mathrm{COPD}$ patients and 20 control smokers, the main findings are as follows:

- As compared to controls, COPD patients exhibited significantly decreased LV deformation properties.

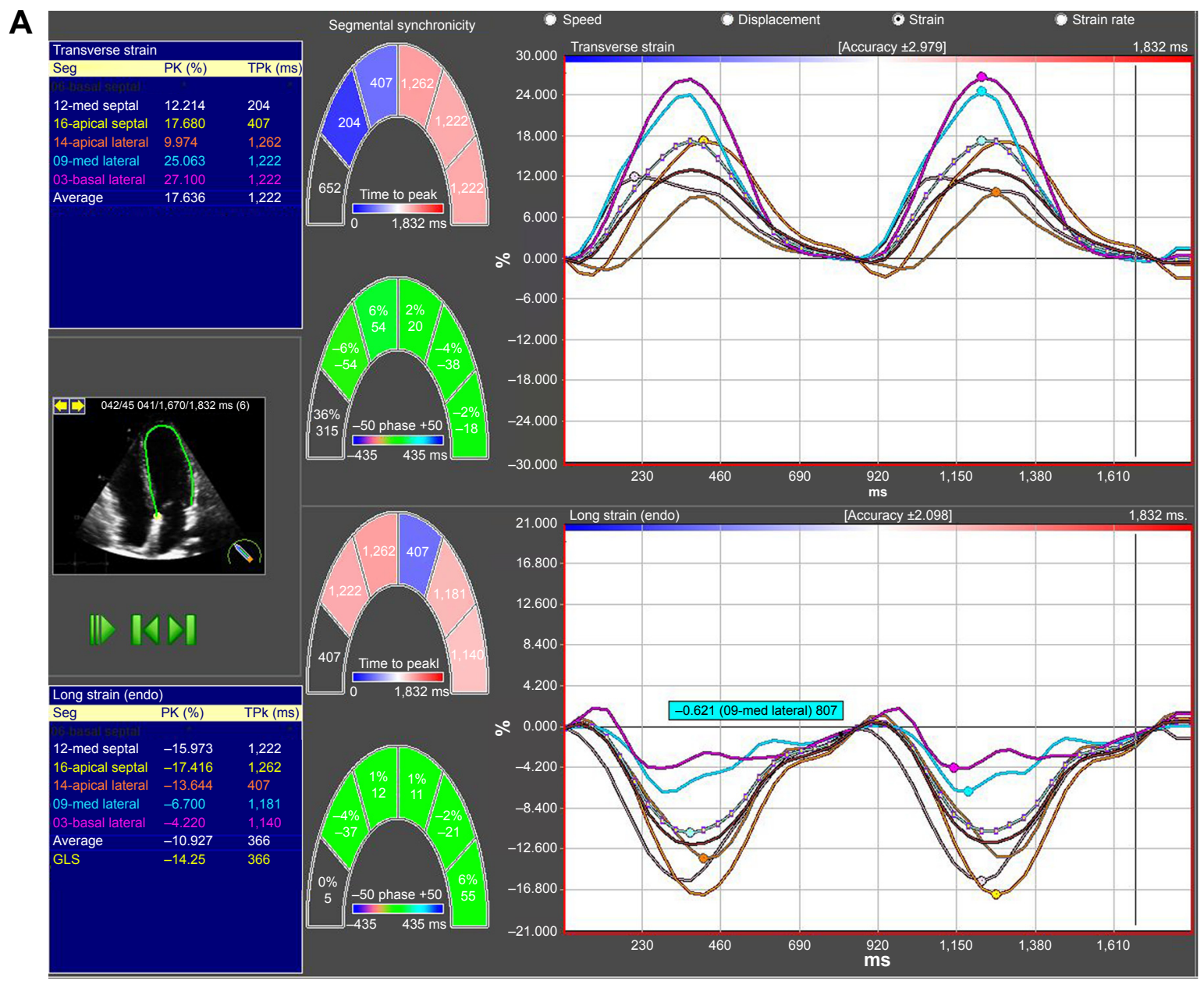

Figure 2 (Continued) 
B

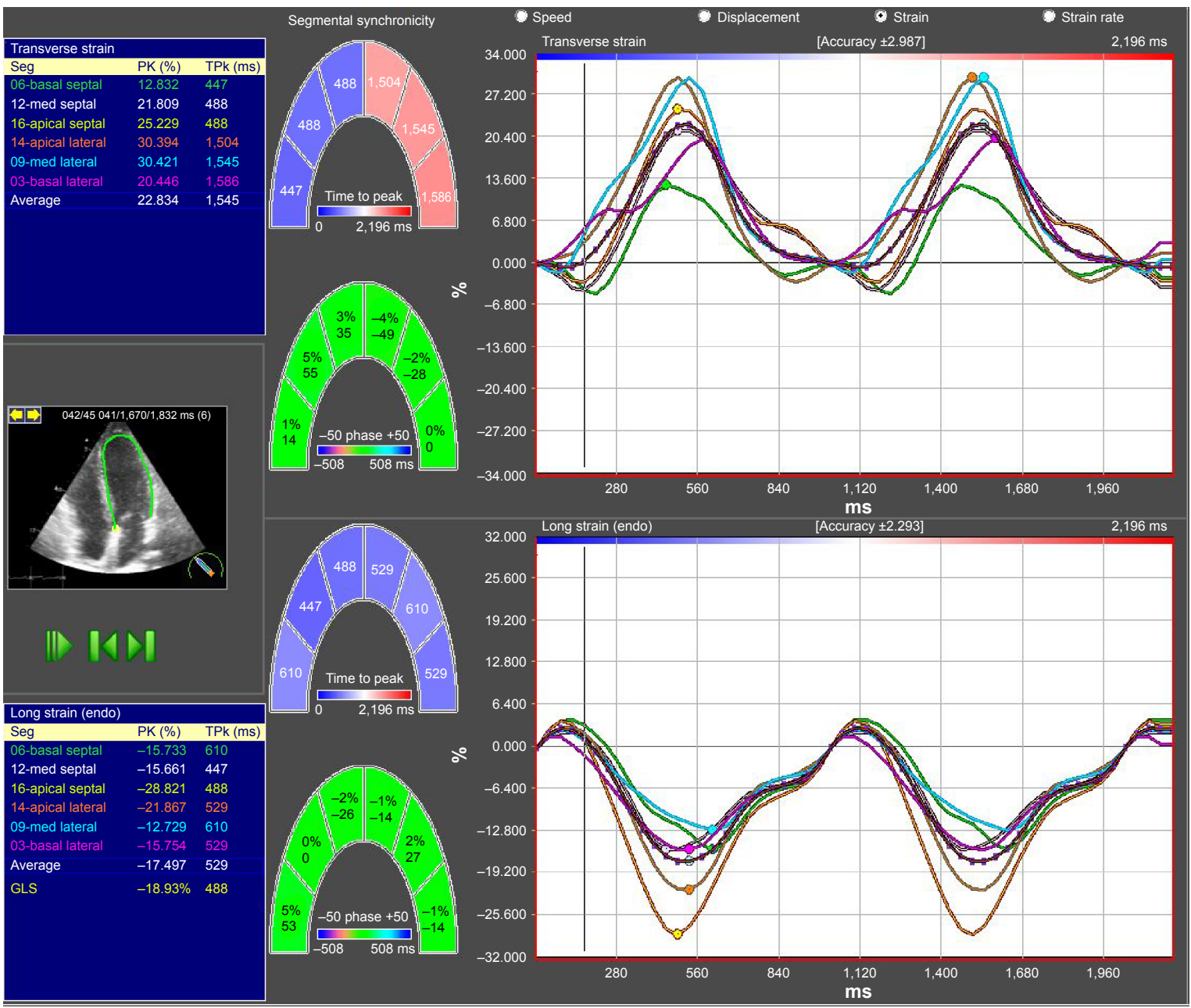

Figure 2 Left ventricular longitudinal strain analysis, visualized by two-dimensional speckle tracking echocardiography.

Notes: Deformation imaging of the left ventricle was performed in both COPD patients $(\mathbf{A})$ and controls (B), which showed significant differences in global and apical longitudinal deformation properties.

Abbreviations: Long, longitudinal; Max, maximum; Seg, segments; PK, peak; TPk, time to peak; GLS, global longitudinal strain; endo, endocardial.

A

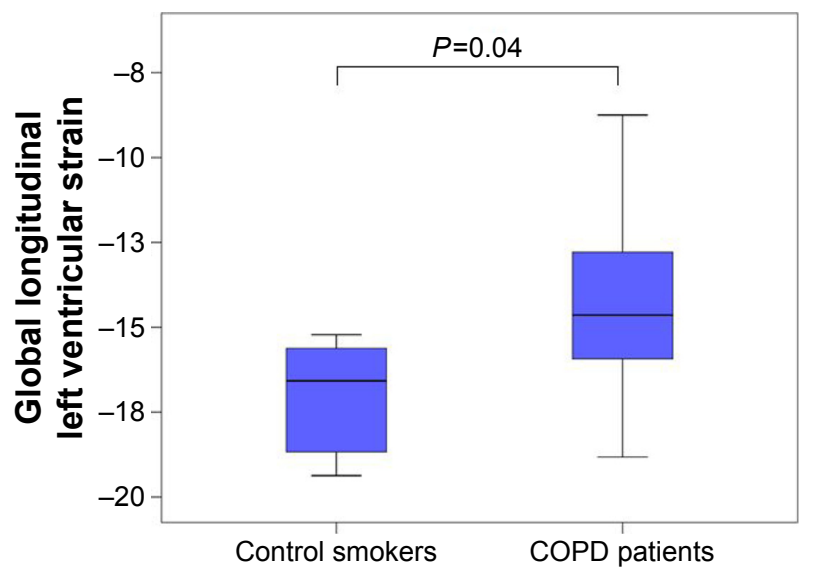

B

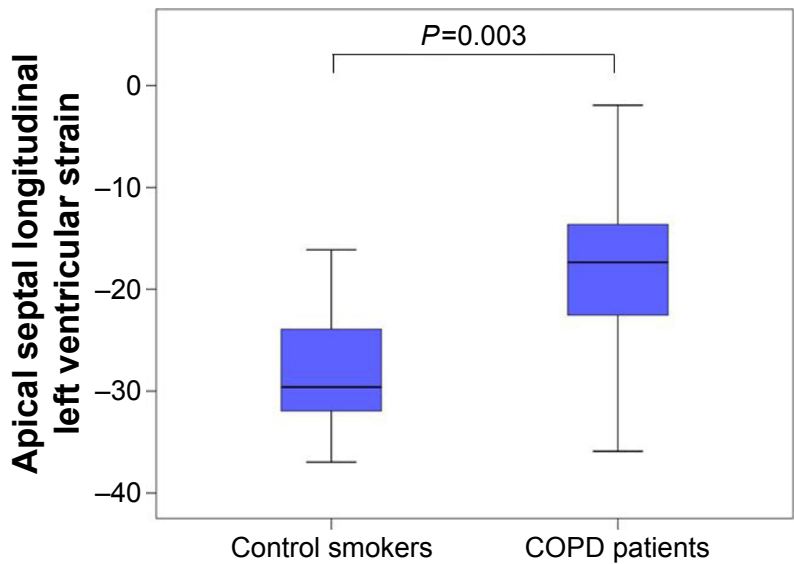

Figure 3 Intercohortal comparison between COPD patients and control smokers for global (A) and apical septal and (B) longitudinal left ventricular strain. 


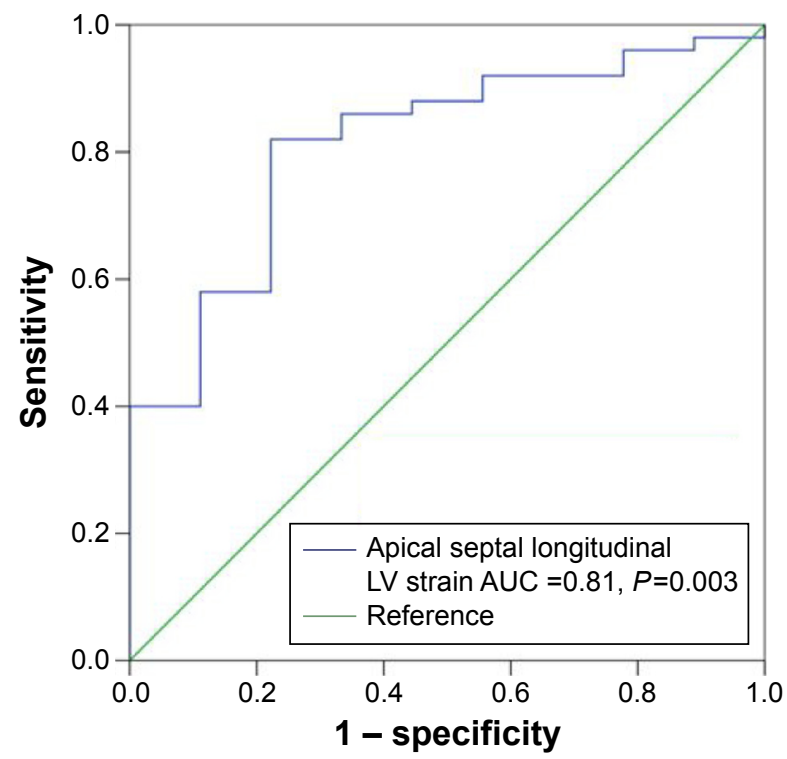

Figure 4 Diagnostic accuracy of apical septal longitudinal LV strain for identification of patients with COPD.

Abbreviations: AUC, area under the curve; LV, left ventricular.

- Regional LV strain, concretely the apical septal longitudinal strain, manifested substantial impairment over COPD GOLD stages.

- The prevalence of sleep apnea was not higher in patients with versus without COPD, nor did the prevalence vary through COPD GOLD stages.

- $5.9 \%$ of COPD patients manifested overlapping OSA that solely correlated with nocturnal ODI, whereas LV performance status was unrelated to OSA occurrence.

In line with the current guidelines, the presence of largely fixed airflow obstruction represents the prime descriptor of

A

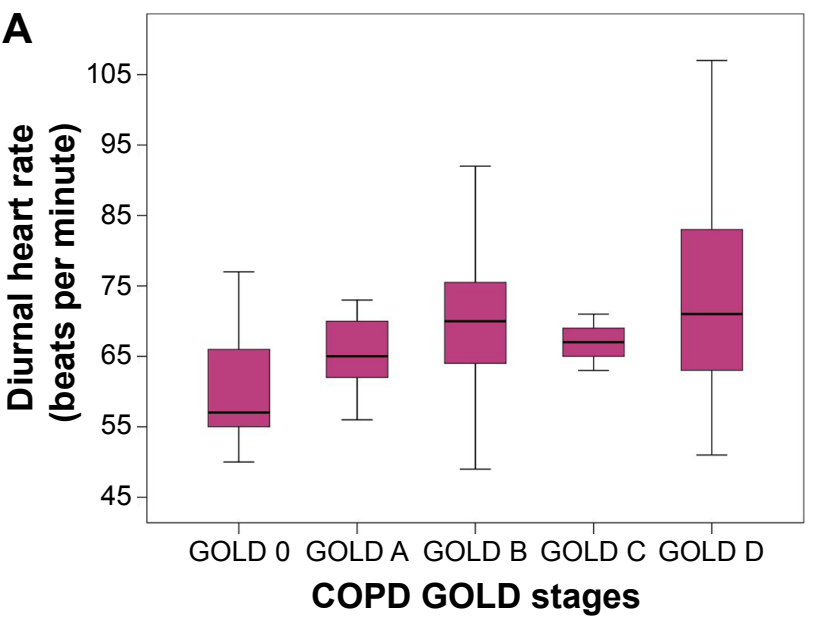

COPD. ${ }^{2}$ Nonetheless, a growing body of evidence indicates that the concept of COPD as a disorder solely restricted to the lungs can no longer be upheld. ${ }^{18}$ As epidemiological data irrefutably demonstrate, the majority of COPD patients die from cardiovascular disease, followed by lung cancer, ${ }^{19}$ whereas the latter one represents the commonest cause of death among patients with mild COPD. ${ }^{2,20}$ Within the cardiovascular spectrum, ischemic heart disease and LV heart failure show major affliction and substantially impact the natural course of COPD. ${ }^{21}$ As to chronic heart failure, more than $20 \%$ of COPD patients have coexisting LV dysfunction. ${ }^{22}$ However, timely diagnosis and effective monitoring of the different disease entities are frequently impeded by their common cardinal symptom of dyspnea. The pathophysiological mechanisms underlying the coexistence of COPD and cardiovascular disorders transcend the shared risk factor and are primarily deducible from the chronic systemic inflammatory processes beyond these disease entities. ${ }^{23}$ Even in stable COPD courses, low-grade systemic inflammation is detectable and is accompanied by blood leukocytosis, as well as increased plasma levels of C-reactive protein, fibrinogen, and inflammatory cytokines. During acute COPD exacerbation, this systemic proinflammatory status flames up and declines in a protracted manner during recovery. ${ }^{24}$ Irrespective of its underlying etiology, chronic heart failure is associated with inflammatory activation. As hypothesized, proinflammatory events may promote cytokine release, and thereby subsequently accelerate LV functional deterioration and heart failure progression. ${ }^{25}$

To evaluate to what extent COPD and LV heart failure mutually exert a negative impact, we presently performed

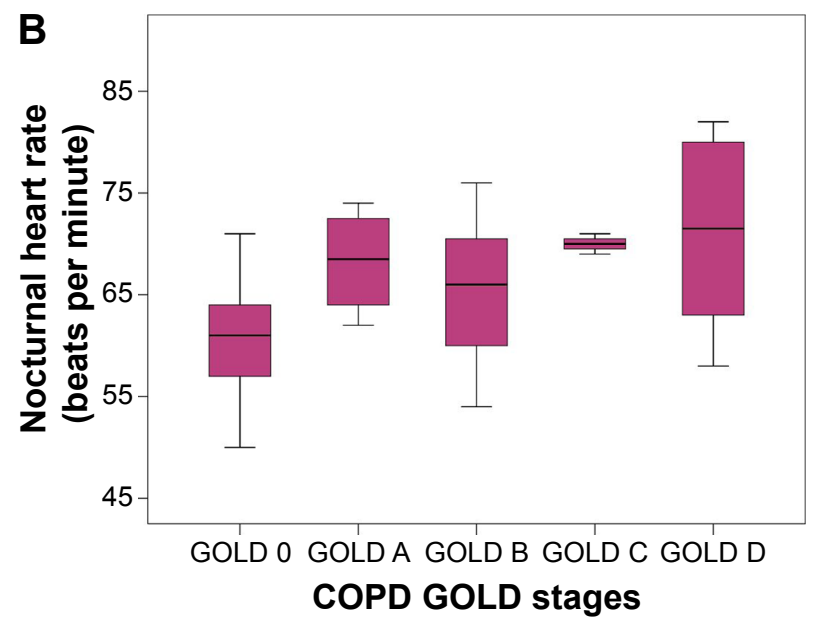

Figure 5 Both diurnal heart rate (A), measured by I2-lead electrocardiogram, and nocturnal heart rate (B), recorded by somnological screening, offer significant increase over COPD GOLD stages $(P=0.01$ and $P=0.00$ I, respectively).

Note: Controls are referred to as "GOLD 0".

Abbreviation: GOLD, Global initiative for Chronic Obstructive Lung Disease. 
conventional echocardiography and STE-based longitudinal deformation imaging. Whereas standard echocardiographic LV indices offered no intercohortal differences, STE-based LV strain analysis identified the global, apical septal, and apical lateral strain to be significantly impaired in COPD patients compared to control smokers, though the prevalence of LV valvular disorders and prediagnosed coronary heart disease did not vary between both groups (Tables 1 and 3). These findings complement the observation made by Schoos et al who performed longitudinal follow-up of 90 COPD patients and identified the global longitudinal LV strain to be the only independent predictor of all-cause long-term mortality. In line with our results, global longitudinal strain did not relevantly vary through COPD GOLD stages. ${ }^{26}$ Recently, a cross-sectional study reported significant global longitudinal LV strain deterioration in COPD patients compared to controls; moreover, global strain was inversely related to BODE index. ${ }^{10}$ Contrary to the aforementioned study, our control cohort was matched against the COPD cohort not only for age and sex, but also for smoking habits including cumulative, pack-year defined smoking quantity. So, we intended to define the impact of mere smoking status on echocardiographic performance. Also, we presently expanded the strain analysis by regional contractility assessment and ascertained the apical septal strain to be not only impaired in COPD patients, but also to correlate with the severity of COPD. Though echo-graded diastolic dysfunction has repeatedly been ascertained in COPD patients, but does not exhibit rising prevalence over COPD GOLD stages, ${ }^{27}$ we presently did not observe significant differences in diastolic function, either within COPD severity degrees or in comparison with controls (Table 3). However, we observed a significant impairment in LV longitudinal strain that can primarily be ascribed to a reduction in apical septal LV strain. The alteration in LV septal deformation capacity might be traced back to COPD-mediated right ventricular (RV) systolic dysfunction, hypertrophy, and dilation that have been described in COPD patients, even in the absence of pulmonary hypertension or diastolic dysfuntion. ${ }^{28}$ In keeping with this, we presently identified a rising prevalence of right bundle branch block over COPD GOLD stages $(P=0.03)$ (Table 3$)$ that per se attenuates septal deformation properties without affecting diastolic performance status, as assessed by conventional echocardiography. The observation of preferentially apical LV impairment might likewise be ascribed to the RV impact, as the apical RV performance has repeatedly been identified as a sensitive descriptor of the total RV functional status. ${ }^{29,30}$ In the present study, we ascertained a prevalence of $5.9 \%$ of overlap by OSA in patients with COPD; likewise, $5.0 \%$ of controls exhibited OSA, a prevalence rate that did not significantly differ from that obtained in COPD patients. This result is consistent with the observations made in the Sleep Heart Health Study by Sanders et al: the prevalence of OSA was not greater in patients with versus without COPD. ${ }^{31}$ According to the current estimates, the prevalence for OSA and COPD is at least $10 \%$, respectively. However, whether each disorder predisposes to a higher incidence of the other remains undetermined. In the World Health Organization-sponsored Multinational Monitoring of Trends and Determinants in Cardiovascular Disease II project, Bednarek et al ascertained the prevalence of overlap syndrome to be $9.2 \% ;^{32}$ however, these data are opposed to the prevalence reported by Soler et al who identified OSA (defined by an AHI $>5 / \mathrm{h}$ ) in $65.9 \%$ of COPD patients. ${ }^{33}$ These diverging results might be traced back to a different diagnostic approach: contrary to Soler et al, we defined presence of sleep apnea by an AHI $\geq 15 / \mathrm{h}$, or an AHI $=5-15 / \mathrm{h}$ along with an ESS $\geq 10$. As reported by Steveling et al, ${ }^{34}$ body mass index turned out to be a major predictor of OSA among COPD patients. In our study, mean BMI accounted for $25.9 \pm 5.4 \mathrm{~kg} / \mathrm{m}^{2}$ among the COPD cohort and might have attenuated the presently observed prevalence of overlapping OSA.

Except for ODI-defined, sleep-related hypoxemia, concomitant OSA did not presently pose the risk of additional cardiac dysfunction to COPD patients: conventional echocardiographic parameters, STE-based strain values, or NT-proBNP levels did not diverge between COPD patients with and without overlapping OSA. Our observations are somewhat inconsistent with the findings obtained by Marin et al who ascertained an increased mortality rate in overlap syndrome patients compared to that in patients with COPD alone, which included a higher number of cardiovascular deaths. ${ }^{35}$ This discrepancy might be attributable both to the limited number of patients identified to suffer from concomitant OSA in our study and to the moderate OSA severity degree among our overlap patients who exhibited a mean AHI of $20.2 \pm 9.5 / \mathrm{h}$. In keeping with this, we observed a higher rate of hypoxemic events only during nighttime, whereas diurnal oxygenation did not differ between groups and might therefore have cushioned the hypoxemia-driven effects on endothelial dysfunction and atherosclerotic processes.

There are several methodological limitations to be considered. First, we have reported a single-center experience and cannot exclude selection or procedure bias. The restricted number of patients enrolled, particularly those exhibiting overlapping OSA, might limit generalizability of study 
results. Also, the observed diurnal and nocturnal heart rate acceleration over COPD severity degrees might have resulted from the sympathetic side effect profile of bronchodilator medication use. Finally, SDB screening was conducted using the two-channel overnight in-home portable SOMNOcheck micro device that relies on automatic data processing and might confer minor diagnostic accuracy than manual scoring like attended in-laboratory polysomnography. As a consequence of the employed screening method, nocturnal oxygenation report did not comprise information on the cumulative percentage of time spent on oxygen saturation below 90\% (CT 90\%). Taken together, COPD itself seems to be accompanied with decreased LV deformation properties that worsen over COPD severity stages, but do not vary in case of overlapping OSA.

\section{Disclosure}

The authors report no conflicts of interest in this work.

\section{References}

1. Lopez AD, Mathers CD, Ezzati M, Jamison DT, Murray CJ. Global and regional burden of disease and risk factors, 2001: systematic analysis of population health data. Lancet. 2006;367(9524):1747-1757.

2. Global Strategy for the Diagnosis, Management and Prevention of COPD, Global Initiative for Chronic Obstructive Lung Disease (GOLD) 2016. Available from: http://www.goldcopd.org/guidelines-globalstrategy-for-diagnosis-management.html. Accessed March 15, 2016.

3. Anthonisen NR. Lessons from the Lung Health Study. Proc Am Thorac Soc. 2004;1(2):143-145.

4. Schünemann HJ, Dorn J, Grant BJ, Winkelstein W Jr, Trevisan M. Pulmonary function is a long-term predictor of mortality in the general population: 29-year follow-up of the Buffalo Health Study. Chest. 2000; 118(3):656-664.

5. Sin DD, Man SF. Why are patients with chronic obstructive pulmonary disease at increased risk of cardiovascular diseases? The potential role of systemic inflammation in chronic obstructive pulmonary disease. Circulation. 2003;107(11):1514-1519.

6. Gan WQ, Man SF, Senthilselvan A, Sin DD. Association between chronic obstructive pulmonary disease and systemic inflammation: a systematic review and a meta-analysis. Thorax. 2004;59(7):574-580.

7. Macchia A, Rodriguez Moncalvo JJ, Kleinert M, et al. Unrecognised ventricular dysfunction in COPD. Eur Respir J. 2012;39(1):51-58.

8. Sabit R, Bolton CE, Fraser AG, et al. Sub-clinical left and right ventricular dysfunction in patients with COPD. Respir Med. 2010;104(8): 1171-1178.

9. Blessberger H, Binder T. Non-invasive imaging: two dimensional speckle tracking echocardiography: basic principles. Heart. 2010;96(9): 716-722.

10. Kalaycioğlu E, Gökdeniz T, Aykan AÇ, et al. Evaluation of left ventricular function and its relationship with multidimensional grading system (BODE index) in patients with COPD. COPD. 2015;12(5):568-574.

11. Flenley DC. Sleep in chronic obstructive lung disease. Clin Chest Med. 1985;6(4):651-661.

12. Ip MS, Tse HF, Lam B, Tsang KW, Lam WK. Endothelial function in obstructive sleep apnea and response to treatment. Am J Respir Crit Care Med. 2004;169(3):348-353.

13. Morgenstern C, Randerath WJ, Schwaibold M, Bolz A, Jané R. Feasibility of noninvasive single-channel automated differentiation of obstructive and central hypopneas with nasal airflow. Respiration. 2013;85(4): 312-318.
14. Sommermeyer D, Zou D, Grote L, Hedner J. Detection of sleep disordered breathing and its central/obstructive character using nasal cannula and finger pulse oximeter. $J$ Clin Sleep Med. 2012;8(5):527-533.

15. Berry RB, Budhiraja R, Gottlieb DJ, et al; American Academy of Sleep Medicine. Rules for scoring respiratory events in sleep: update of the 2007 AASM Manual for the Scoring of Sleep and Associated Events. Deliberations of the Sleep Apnea Definitions Task Force of the American Academy of Sleep Medicine. J Clin Sleep Med. 2012;8(5): 597-619.

16. Johns MW. A new method for measuring daytime sleepiness: the Epworth sleepiness scale. Sleep. 1991;14(6):540-545.

17. American College of Cardiology Foundation Appropriate Use Criteria Task Force; American Society of Echocardiography; American Heart Association; American Society of Nuclear Cardiology; Heart Failure Society of America; Heart Rhythm Society; Society for Cardiovascular Angiography and Interventions; Society of Critical Care Medicine; Society of Cardiovascular Computed Tomography; Society for Cardiovascular Magnetic Resonance; American College of Chest Physicians; Douglas PS, Garcia MJ, Haines DE, et al. ACCF/ASE/AHA/ASNC/ HFSA/HRS/SCAI/SCCM/SCCT/SCMR 2011 appropriate use criteria for echocardiography. A report of the American College of Cardiology Foundation Appropriate Use Criteria Task Force, American Society of Echocardiography, American Heart Association, American Society of Nuclear Cardiology, Heart Failure Society of America, Heart Rhythm Society, Society for Cardiovascular Angiography and Interventions, Society of Critical Care Medicine, Society of Cardiovascular Computed Tomography, Society for Cardiovascular Magnetic Resonance American College of Chest Physicians. J Am Soc Echocardiogr. 2011; 24(3):229-267.

18. Nussbaumer-Ochsner Y, Rabe KF. Systemic manifestations of COPD. Chest. 2011;139(1):165-173.

19. Hansell AL, Walk JA, Soriano JB. What do chronic obstructive pulmonary disease patients die from? A multiple cause coding analysis. Eur Respir J. 2003;22(5):809-814.

20. Anthonisen NR, Connett JE, Enright PL, Manfreda J; Lung Health Study Research Group. Hospitalizations and mortality in the Lung Health Study. Am J Respir Crit Care Med. 2002;166(3):333-339.

21. Mannino DM, Thorn D, Swensen A, Holguin F. Prevalence and outcomes of diabetes, hypertension and cardiovascular disease in COPD. Eur Respir J. 2008;32(4):962-969.

22. Rutten FH, Cramer MJ, Lammers JW, Grobbee DE, Hoes AW. Heart failure and chronic obstructive pulmonary disease: An ignored combination? Eur J Heart Fail. 2006;8(7):706-711.

23. Maclay JD, MacNee W. Cardiovascular disease in COPD: mechanisms. Chest. 2013;143(3):798-807.

24. Ukena C, Mahfoud F, Kindermann M, et al. The cardiopulmonary continuum systemic inflammation as 'common soil' of heart and lung disease. Int J Cardiol. 2010;145(2):172-176.

25. Seta Y, Shan K, Bozkurt B, Oral H, Mann DL. Basic mechanisms in heart failure: the cytokine hypothesis. J Card Fail. 1996;2(3):243-249.

26. Schoos MM, Dalsgaard M, Kjærgaard J, et al. Echocardiographic predictors of exercise capacity and mortality in chronic obstructive pulmonary disease. BMC Cardiovasc Disord. 2013;13:84.

27. Huang YS, Feng YC, Zhang J, et al. Impact of chronic obstructive pulmonary diseases on left ventricular diastolic function in hospitalized elderly patients. Clin Interv Aging. 2014;10:81-87.

28. Hilde JM, Skjørten I, Grøtta OJ, et al. Right ventricular dysfunction and remodeling in chronic obstructive pulmonary disease without pulmonary hypertension. J Am Coll Cardiol. 2013;62(12): $1103-1111$

29. Fernandez-Friera L, Garcia-Alvarez A, Guzman G, et al. Apical right ventricular dysfunction in patients with pulmonary hypertension demonstrated with magnetic resonance. Heart. 2011;97(15): $1250-1256$.

30. Pizarro C, Schueler R, Hammerstingl C, Tuleta I, Nickenig G, Skowasch D. Impact of endoscopic lung volume reduction on right ventricular myocardial function. PLoS One. 2015;10(4):e0121377. 
31. Sanders MH, Newman AB, Haggerty CL, et al; Sleep Heart Health Study. Sleep and sleep-disordered breathing in adults with predominantly mild obstructive airway disease. Am J Respir Crit Care Med. 2003; 167(1):7-14.

32. Bednarek M, Plywaczewski R, Jonczak L, Zielinski J. There is no relationship between chronic obstructive pulmonary disease and obstructive sleep apnea syndrome: a population study. Respiration. 2005;72(2): $142-149$.

33. Soler X, Gaio E, Powell FL, et al. High prevalence of obstructive sleep apnea in patients with moderate to severe chronic obstructive pulmonary disease. Ann Am Thorac Soc. 2015;12(8):1219-1225.
34. Steveling EH, Clarenbach CF, Miedinger D, et al. Predictors of the overlap syndrome and its association with comorbidities in patients with chronic obstructive pulmonary disease. Respiration. 2014;88(6): 451-457.

35. Marin JM, Soriano JB, Carrizo SJ, Boldova A, Celli BR. Outcomes in patients with chronic obstructive pulmonary disease and obstructive sleep apnea: the overlap syndrome. Am J Respir Crit Care Med. 2010;182(3): 325-331.

\section{Publish your work in this journal}

The International Journal of COPD is an international, peer-reviewed journal of therapeutics and pharmacology focusing on concise rapid reporting of clinical studies and reviews in COPD. Special focus is given to the pathophysiological processes underlying the disease, intervention programs, patient focused education, and self management protocols.

\section{Dovepress}

This journal is indexed on PubMed Central, MedLine and CAS. The manuscript management system is completely online and includes a very quick and fair peer-review system, which is all easy to use. Visit $\mathrm{http}: / / \mathrm{www}$.dovepress.com/testimonials.php to read real quotes from published authors. 